\title{
Transient Optical-Microwave Spectroscopy for Electron Mobility Assessment in Solids and Gels: A Comprehensive Approach
}

\author{
Wakana Matsuda ${ }^{1}$, Tsuneaki Sakurai ${ }^{1}$, Goutam Ghosh ${ }^{2}$, Suhrit Ghosh $^{2 *}$, and Shu Seki ${ }^{1 * *}$ \\ ${ }^{1}$ Department of Molecular Engineering, Graduate School of Engineering, \\ Kyoto University, Nishikyo-ku, Kyoto 615-8510, Japan \\ ${ }^{2}$ Polymer Science Unit, Indian Association for the Cultivation of Science, \\ Mullick Road, Kolkata, 700032, India \\ *psusg2@iacs.res.in \\ **seki@moleng.kyoto-u.ac.jp
}

\begin{abstract}
Organic small molecular semiconductors have been promising candidate for the future electronic materials and devices, and their charge transport potentials have been expected to catch-up and surpass the benchmark one in $\mathrm{Si}$ - the giant in the semiconductor materials. As represented in the mobility values observed in graphene with $\mu>10^{4} \mathrm{~cm}^{2} \mathrm{~V}^{-1} \mathrm{~s}^{-1}$, the extended $\pi$-conjugated electron systems provide highly conductive platforms for electrons and holes, however the charge transport in aggregates of conjugated small molecules is disturbed by inter-molecular thermal fluctuations, resulting in the lower mobility of charge carriers in the molecular systems. We have developed a novel assessment technique of charge carrier mobility on the nanoscale molecular aggregates, referred to as time-resolved microwave conductivity (TRMC) measurement, where the non-contact approach minimizes the inter-aggregates issues in the conventional assessment techniques. Particularly, the combination of TRMC with transient absorption spectroscopy (TAS) realizes herein fully experimental non-contact measurement of mobility in supramolecular architectures without structural modulation of the archtectures in the device fabrication protocols. Electron mobility observed in one-dimensional naphthalenediimide (NDI) stacking structures was determined as $\mu, 1 \mathrm{D}=10^{-3}-10^{-4} \mathrm{~cm}^{2} \mathrm{~V}^{-1} \mathrm{~s}^{-1}$ based on the values of photoconductivity $\Delta \sigma$ and photo-carrier generation yield $\phi$ determined by TRMC and TAS, respectively. The NDI stacked structures with a variety of inter-molecular interactions were systematically examined by the combined spectroscopy systems, revealing the remarkable impact of interactions stabilizing the stacking structures on the mobility values.
\end{abstract}

Keywords: Microwave conductivity, Transient absorption, Electron mobility, Supramolecular architecture, Gel

\section{Introduction}

Conjugated organic molecular motifs have been designed and developed day-by-day, and the variation of the chemical structures with developed conjugated electron systems has been dramatically increased particularly in this decade [1-3]. The most important primary parameter reflecting the potentials of conjugated organic molecular materials is the mobility of charge carriers in their practical application to organic electronic devices. Till date, the techniques for the assessment of the mobility values have been represented as the field- effect-transistor (FET) [4,5], time-of-flight (TOF) [6-8], space-charge-limited current (SCLC) $[9,10]$, and impedance spectroscopy (IS) $[11,12]$ methods. However, all the above techniques require the fabrication of transistor or capacitor -like device structures, as well as the subsequent device optimization to deduce and mark the highest and intrinsic value of mobility. The long and winding protocols for the optimization are hampering to match up the assessment throughput with the increasing variation of the materials newly synthesized and reported in these days. This 
implies the most important requisite for the development of molecular electronics is the rapid and accurate screening of the materials, and thus the non-contact measurements of optical conductivity have been often the choices to lead the intrinsic potentials of the materials. Optical conductivity measurements [13-20] are based on the dielectricloss mechanisms of electromagnetic waves with the matters, hence with the mobile electrons therein. The local motion of electron absorbs significantly the power of the probing microwaves via inducing local and oscillating motion of the electrons. The dielectric loss of the microwave depends strongly on the frequency of microwaves employed, and the choice of the frequency is particularly important for the accurate detection of the motion, and eventually estimate the mobility [15-20] Assuming the typical sizes of molecules as $1 \mathrm{~nm}$ and the value of mobility $(\mu)$ observed in the representative organic semiconducting molecules as $\mu \sim 10^{-2}-1 \mathrm{~cm}^{2} \mathrm{~V}^{-1} \mathrm{~s}^{-1}$, the relaxation time of the charge carrier in the media is in the range of ps, suggesting an appropriate selection of the frequency of the probing electromagnetic waves in $\mathrm{GHz}$ regime to take effects of inter-molecular electron transport into accounts. The electromagnetic waves, often referred to as microwaves, are herein employed for the measurement which has been applied for the measurement of mobility in conjugated polymers (intra-chain mobility) [21-29], conjugated organic supramolecular architectures [30-40], molecular crystals [41-45], conjugated organic frameworks [49-51], etc.

The critical issue in the assessment of mobility is in the fair and rapid measurement of charge carrier density injected into the materials. The carrier injection has been realized by radiolysis [15-17], photolysis [18,22-52], electric field [44,45,53-59], and chemical doping. The radiolysis of the molecular system provides the direct and precise determination of the yield of charge carriers injected into the materials via $G$-values of ionization depending only on the electron density of the matters, which has been investigated for a variety of organic media and the databases is already in our hands. However, the pulsed radiation sources with high enough energy to ionize the molecules are in the limit of use, and photoionization with the pulsed laser system is the second choice in view of availability. Photoionization is also the cases of fundamental processes in the practical devices of organic photovoltaics $(\mathrm{OPVc})$ and organic light emitting diodes (OLED), thus it is also advantageous to correlate the derived photoconductivity and mobility of charge carriers with the overall device performances [60-64]. Here, the non-contact experimental determination of the photoionization yield [65-68] plays an important role for the determination of mobility, and realizing fully non-contact protocols in the assessment. The latter is crucial especially in case of organic supramolecular architectures because the device fabrication with electrodes attached to organic media often causes the severe damages of the materials structures as well as the interfacial ones where the major charge carrier pathways present.

Herein we report on the fully experimental protocols to deliver the mobility of electron in the conjugated organic gels: the most "soft" materials platforms. Designing the architectures with the pathways for n-type electron transport is the most taxing steps in the organic electronics, and the protocols was successfully applied for the assessment via the combination of time-resolved microwave conductivity (TRMC) and transient absorption spectroscopy (TAS) measurements [1820,22-52,69].

\section{Experimental}

2.1. Materials and general methods

$n$-Decane, methylcyclohexane (MCH), chloroform, and tetrahydrofuran (THF) were purchased from Wako Co., Ltd., or Tokyo Chemical Industry Co., Ltd., and used as received. Tetrabutylammonium cyanide (TBACN) was purchased from Sigma-Aldrich Co. and used without further purification. cNDI-1 was synthesized according to the literature [70] with modification. Electronic absorption spectra were recorded on a JASCO V-570 UV/VIS/NIR spectrophotometer

Flash-photolysis (FP)-TRMC measurements were performed at room temperature under air. The samples for FP-TRMC were prepared as described below. A stock solution of cNDI-1 (4.0 or $10 \mathrm{mM}$ ) was prepared in chloroform and sonicated about $20 \mathrm{sec}$. A desired amount of the stock solution was taken to a vial and dried by a heat gun to get a thin film. A desired concentration (0.1 $\mathrm{mM}$ or $4.0 \mathrm{mM}$ ) of a low solubility solvent was added into the vial, and the mixture was heated by a heat gun until it became the homogeneous solution. The color was changed from dark violet to magenta. Then the solution was cooled to room temperature and used for the measurement. A $4.0 \mathrm{mM}$ decane 
(a)

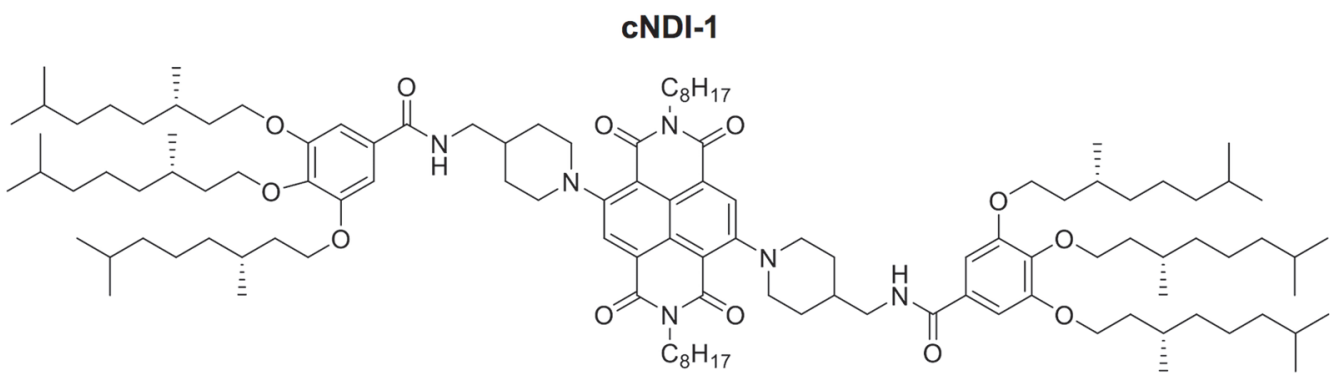

(b)

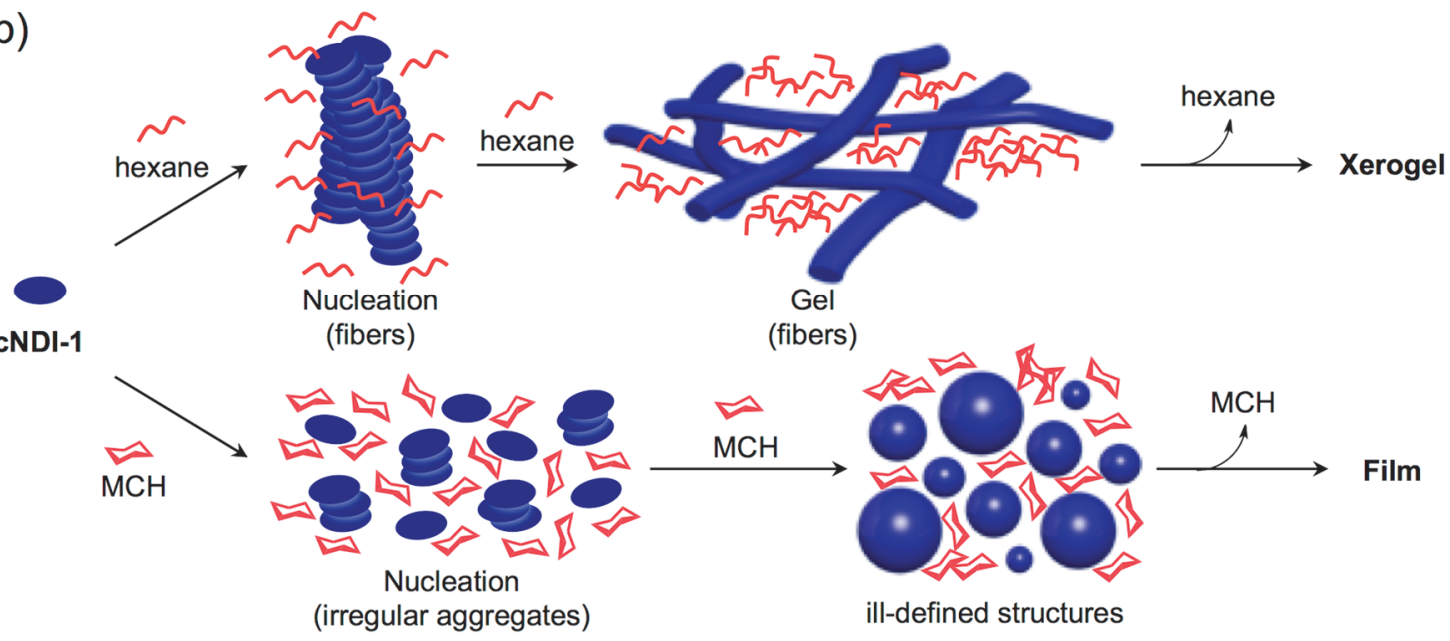

Fig. 1. (a) Chemical structure of cNDI-1 and (b) possible schematic illustration of formation of gel in decane and illdefined structures in methylcyclohexane for cNDI-1.

solution was stored r.t. overnight to form a gel state. For FP-TRMC, the solutions were dropcast on a quartz substrate and dried. The gel was mounted on a quartz without further treatment, and a xerogel state was obtained after drying a gel state at r.t. The substrate is loaded into a TE102 mode microwave cavity. Transient charge carriers were photo-generated upon excitation to laser pulses of third harmonic generation $(\lambda=355 \mathrm{~nm})$ from a Spectra Physics INDI-HG Nd:YAG laser with a pulse duration of 5-8 ns. The photon density of a $355 \mathrm{~nm}$ pulse, operated at $10 \mathrm{~Hz}$, was $9.1 \times 10^{15}$ photons $\mathrm{cm}^{-2}$ pulse ${ }^{-1}$. The probing microwave frequency and power were set at $9.1 \mathrm{GHz}$ and $3 \mathrm{~mW}$, respectively. Photoconductivity transients, demodulated through a GaAs crystal-diode with Schottky-barriers (rise time $<1 \mathrm{~ns}$ ), were monitored by a Tektronix TDS3032B digital oscilloscope. The observed conductivities were normalized, given by a photocarrier generation yield $(\phi)$ multiplied by sum of the charge carrier mobilities $(\Sigma \mu)$, according to the equation,

$\phi \Sigma \mu=\mathrm{A} \cdot\left\{e I_{0} F_{L}\right\}^{-1} \cdot\left(\Delta P_{r} / P_{r}\right)$

where, $e, \mathrm{~A}, I_{0}, F_{\mathrm{L}}, P_{\mathrm{r}}$, and $\Delta P_{\mathrm{r}}$ represent elementary charge, sensitivity factor $\left(\mathrm{S} \mathrm{cm}^{-1}\right)$, incident photon density of the excitation laser (photon $\mathrm{cm}^{-2}$ ), correction factor $\left(\mathrm{cm}^{-1}\right)$, and reflected microwave power and its change, respectively.

Transient absorption spectroscopy (TAS) was carried out by using the identical films used for FPTRMC measurements. The YAG laser was used as a pump light source, where the photon density of a $355 \mathrm{~nm}$ pulse was $9.1 \times 10^{15}$ photons $\mathrm{cm}^{-2}$ pulse 1. A white light continuum from a Xe lamp was used as a probe light source. The monochromated probe light was guided into a Hamamatsu C7700 wide-dynamic-range streak camera system, which collected a two-dimensional image of the spectral and temporal profiles of light intensity. The equation for the determination of mobility values is described in the following section.

\section{Results and discussion}

Molecular systems with NDI cores have been expected to provide pathways for electrons in their aggregated forms, particularly along the stacking axes orthogonal to the planar structures of the cores [71-76]. Diimide structures lower the energy levels of the lowest unoccupied molecular orbitals (LUMOs), and this is the case giving the high stability of electrons on the molecular core: the radical anions. Perylenediimides (PDIs) are the 
similar $\pi$-conjugated cores with planar structures [77-80] and the lower LUMOs, and unlikely to the case of NDI, the level of HOMO shifts upward and hence the stacking structures of PDIs provides not only the pathways for electrons but also for holes (radical cations). NDI cores have been often the choices as the key molecular motifs to promote supramolecular gels in their solutions via the strong inter-molecular interactions facilitated by the substituents and NDI cores, revealing the successful formation of gels in a variety of solvents [34,38,39,81-86]. Note that all the gel structures are primary possessing one-dimensional alignment of NDI cores, suggesting the feasibility of the gels as the conducting materials for electrons.

The chemical structure of cNDI-1 is shown in Fig. 1a. A secondary amine, cyclohexyl amine, was introduced into the NDI core, giving donoracceptor electronic nature to this molecule. As shown in Fig. 2, electronic absorption spectra indicate the broad charge-transfer absorption band at around $600 \mathrm{~nm}$. This molecule has long chiral paraffinic chains at its peripheries, and these peripheral units are linked to the NDI core through amide groups. Due to the hydrogen bonding among the amide groups and $\pi-\pi$ interactions among NDI units, it stacks to form one-dimensional fibers or ill-defined aggregates [70]. The selfassembly pathways are controlled by solvents, where decane triggers ordered nucleation of cNDI1 molecules while $\mathrm{MCH}$ induces irregular nucleation (Fig. 1b) [70]. In decane, the nuclei are the short fibers of cNDI-1s that further grow into supramolecular bundled nanofibers. Under high concentration, assembly of the bundled nanofibers form a partial gel (Fig. 2, inset). On the other hand, the irregular aggregates of cNDI-1 develop into spherical ill-defined structures, in which intermolecular stacking of cNDI- 1 is present. The cast films prepared from decane and $\mathrm{MCH}$ solutions (or partial gels) most likely contain nanostructures of one-dimensional nanofibers and spherical aggregates, respectively. A xerogel film from decane gel of cNDI-1 affords absorption spectrum that is quite similar with its THF solution (Fig. 2).

The tunable nature of the gel structures implies that the structure is sensitive to the temperature and solvents, and the thin solid film based conventional techniques for conductivity and mobility assessment are unfavorable and inapplicable to reveal the conductive nature of the NDI stacking structure as it forms. FP-TRMC with TAS measurements provides totally non-contact protocols: the most appropriate one for the present gel system. Prior to the conductivity measurement, the radical anions are presumed as the predominant conductive species and produced by the one electron reduction of the molecular system with TBACN [87]. The electronic absorption spectrum of the radical anions is shown in Fig. 2, giving a clear signature of NDI anions formed in the present system, and the molar extinction coefficient of $\mathrm{NDI}^{\circ-}$ was determined as $\varepsilon\left(\mathrm{NDI}^{\circ}\right)=3600 \mathrm{~mol}^{-1}$ $\mathrm{dm}^{3} \mathrm{~cm}^{-1}$ at $765 \mathrm{~nm}$. This value is adopted for the calculation of mobility in the present system from the observed conductivity transients.

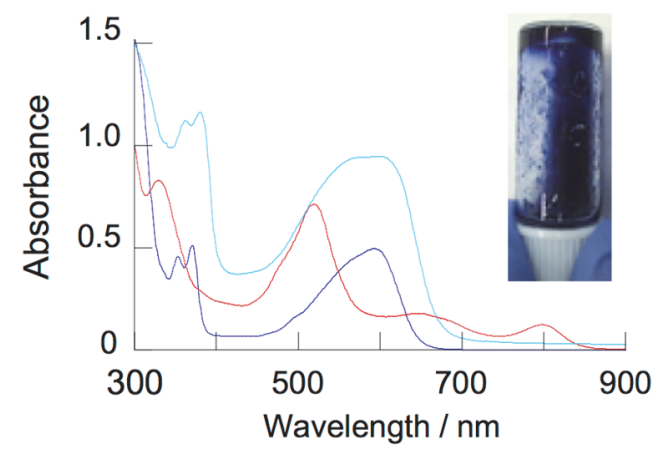

Fig. 2. Electronic absorption spectra of cNDI-1 in THF (blue) at $0.034 \mathrm{mM}$ and its radical anion form (red) generated by addition of TBACN, and a xerogel film (pale blue) prepared from $0.1 \mathrm{mM}$ decane solution. Inset shows a photo graph of partial gel state for cNDI-1 in decane at $4 \mathrm{mM}$.

Dielectric loss factor for the microwave at $9 \mathrm{GHz}$ depends strongly on the dielectric constant of the media, and is roughly estimated from the relative dielectric constant $\varepsilon(\omega)$ at $\omega=0$. For the formation of supramolecular gel structure in the present work, decane, $\mathrm{MCH}$, and $\mathrm{CHCl}_{3}$ were used with the value of $\varepsilon(0)=1.99,4.81$, and 8.93, respectively. The static dielectric loss of probing microwave can be negligible for decane, however those of $\mathrm{MCH}$ and $\mathrm{CHCl}_{3}$ are considerable not to penetrate the microwave into the media with enough electric field strength for TRMC measurements. To address this issue, the combined TRMC-TAS measurement was firstly applied to a xerogel state of cNDI-1 after evaporation of decane $(0.1 \mathrm{mM})$. The film of xerogels deposited onto a quartz substrate were exposed to an excitation light pulses with the duration of $<5 \mathrm{~ns}$ at $355 \mathrm{~nm}$ where the high enough transition dipole of NDI cores was observed as shown in Fig. 2. A two-dimensional image of transient absorption is represented in Fig. 3a with the simultaneously recorded transient spectra (Fig. 3b) and a kinetic trace observed at $765 \mathrm{~nm}$ (Fig. 3c), 

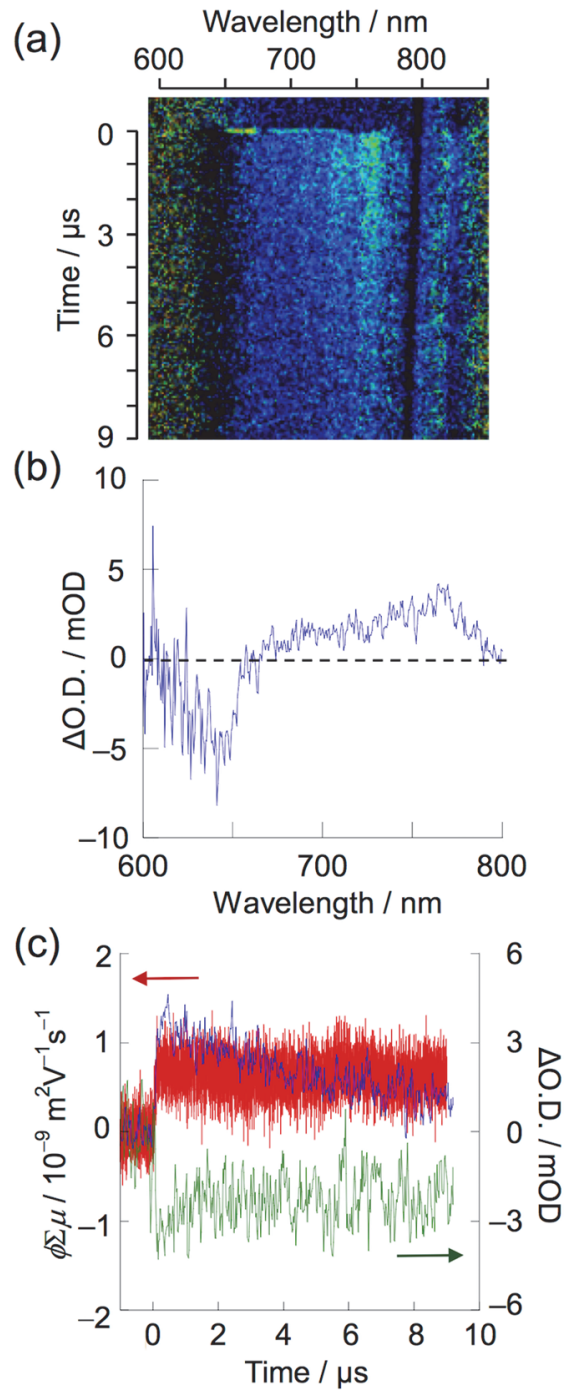

Fig. 3. (a) Two-dimensional transient absorption spectroscopy image and (b) transient optical absorption spectra (averaged over $0.5 \mu \mathrm{s}$ ) for xerogel film of cNDI1 (from $0.1 \mathrm{mM}$ in decane) observed at $0 \mu$ s after pulse irradiation at $355 \mathrm{~nm}$ pulses at $9.1 \times 10^{15}$ photons $\mathrm{cm}^{-2}$. (c) Kinetic traces of transient conductivity (red) and optical absorption at $650 \mathrm{~nm}$ (green) and $765 \mathrm{~nm}$ (blue) observed for xerogel film of cNDI-1 (from $0.1 \mathrm{mM}$ in decane) after pulse irradiation at $9.1 \times 10^{15}$ photons $\mathrm{cm}^{-2}$.

respectively. The clear transient absorption was monitored around 750-800 nm, which was the characteristic transition of $\mathrm{NDI}^{\bullet-}$ supported by both the spectrum in Fig. 2 and the one in previous report [88]. TAS measurements were performed under ambient conditions with the spectral accumulation of $\sim 500$ shot excitations. Photoconductivity transients were recorded for the identical xerogel film as given in Fig. $3 \mathrm{c}$ by FP-TRMC measurement. It should be noted that the kinetic trace exhibits the lifetime over a few $100 \mu$ s (Fig. 4), which is exceptionally long as for the transient species of radical anions unstable against air $\left(\mathrm{O}_{2}\right)$ or environmental $\left(\mathrm{H}_{2} \mathrm{O}\right)$. The 1D stacking structures of NDI cores are stabilized by the interactions (hydrogen bondings) among the amide peripheries and surrounded by the long and expanded alkyl chains, and this is the case hampering the access of even $\mathrm{O}_{2}$ molecules to the conductive core structures.

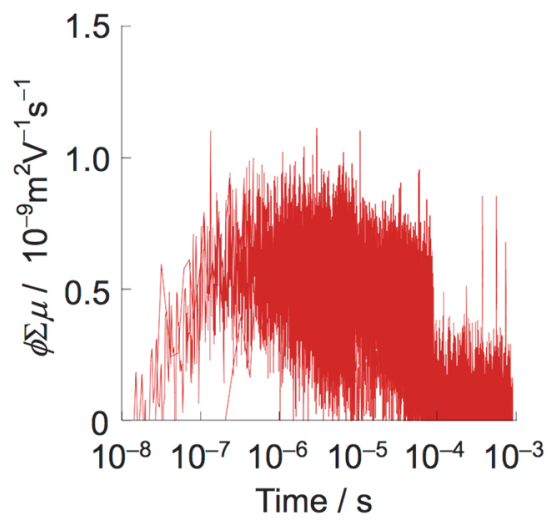

Fig. 4. Kinetic traces of transient conductivity in long time scale observed for xerogel film of cNDI-1 upon exposure to $355 \mathrm{~nm}$ pulses at $9.1 \times 10^{15}$ photons $\mathrm{cm}^{-2}$.

The kinetic traces of both TAS at $765 \mathrm{~nm}$ and TRMC are overlapped, suggesting the radical anions as the major conductive species in the present system. The kinetic overlap allows us to estimate the value of mobility by a fully experimental protocol; TAS provides information of the number density of photo-generated carriers and subsequently quantum efficiency $(\phi)$ of photoionization in the present time regime, and TRMC gives the value of pseudo-conductivity represented as $\phi \Sigma \mu$. Here the optical density ( $\Delta$ O.D.) of $\mathrm{NDI}^{\bullet}$ in the kinetic traces at $1 \mu \mathrm{s}$ after pulse exposure is converted directly into $\phi$ as follows,

$$
\phi=\Delta \mathrm{O} . \mathrm{D} \cdot\left\{\varepsilon\left(\mathrm{NDI}^{-}\right) \cdot I_{0} \cdot F\right\}^{-1}
$$

where $I_{0}$ and $\mathrm{F}$ are the excitation photon density $\left(\mathrm{m}^{-2}\right)$ and the density factor $\left(\mathrm{mol} \mathrm{m} \mathrm{m}^{-2}\right.$ photons $\left.{ }^{-1}\right)$, respectively. Dividing the pseudo-conductivity by $\phi$ gives an estimate of isotropic electron mobility $\left(\mu\right.$ ) on cNDI-1 stacking structures as $\mu_{-}=7 \times 10^{-5}$ $\mathrm{cm}^{2} \mathrm{~V}^{-1} \mathrm{~s}^{-1}$. Assuming the anisotropic 1D stacking structures of cNDI-1 orthogonal to the molecular planes, the mobility of electrons along the stacking axes are deduced to be $\mu_{-, 1 \mathrm{D}}=2 \times 10^{-4} \mathrm{~cm}^{2} \mathrm{~V}^{-1} \mathrm{~s}^{-1}$ [89], which is competitive to the conventional ntype molecular semiconductors in their selfassembled states. 
A partial gel of cNDI-1 was obtained from a concentrated $n$-decane solution $(4 \mathrm{mM})$ after standing at room temperature for several days. The partial gel was deposited onto a quartz substrate and extra solvent was removed by drying under ambient atmosphere, resulting in the gel sample for TRMC/TAS experiments. After the measurements, the gel was completely dried at room temperature overnight, giving the xerogel sample. Transient photoconductivity and optical absorption of both gel and xerogel states are shown in Fig. 5. The xerogel film gave a clear decay of $\mathrm{NDI}^{-}$in TAS and the kinetic traces are completely overlappedwith that of the TRMC in Figs. 5e and 5f. The peak and $\Delta$ O.D. of NDI ${ }^{\circ}$ in TAS measurements in Figs. $5 \mathrm{c}$ and $5 \mathrm{~d}$ are similar to each other and indicated almost equal $\phi$ value of approximately $3 \times$ $10^{-2}$.
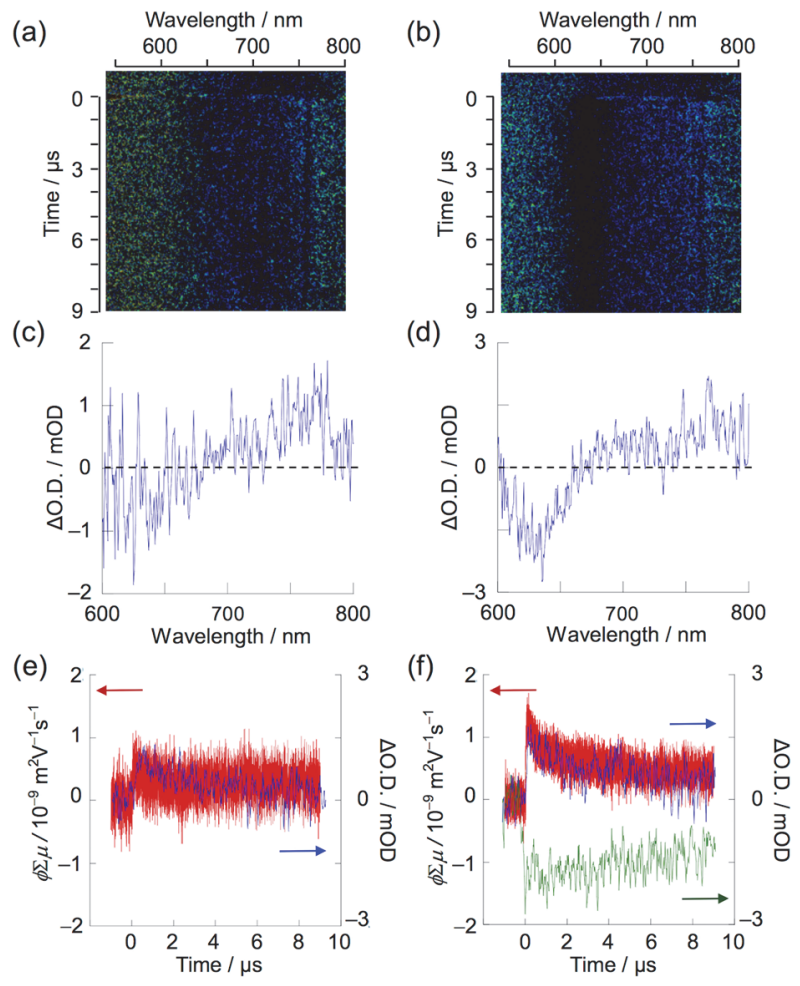

Fig. 5. (a,b) Two-dimensional transient absorption spectroscopy image and $(\mathrm{c}, \mathrm{d})$ transient optical absorption spectra (averaged over $0.5 \mu \mathrm{s}$ ) for $(\mathrm{a}, \mathrm{c})$ gel and $(\mathrm{b}, \mathrm{d})$ xerogel film of cNDI- 1 observed at $0 \mu$ s after pulse irradiation at $355 \mathrm{~nm}$ pulses at $9.1 \times 10^{15}$ photons $\mathrm{cm}^{-2}$. (e,f) Kinetic traces of transient conductivity (red) and optical absorption at $650 \mathrm{~nm}$ (green) and $765 \mathrm{~nm}$ (blue) observed for (e) gel and (f) xerogel film of cNDI- 1 after pulse irradiation at $9.1 \times$ $10^{15}$ photons $\mathrm{cm}^{-2}$.
On the other hand, the photoconductivity of the xerogel state is two times higher than that of the gel state. It means that the mobility is increased after drying the gels up to $\mu=4 \times 10^{-4} \mathrm{~cm}^{2} \mathrm{~V}^{-1} \mathrm{~s}^{-1}$, and thus the one-dimensional mobility is assumed to be $\mu_{-, 1 \mathrm{D}}=1 \times 10^{-3} \mathrm{~cm}^{2} \mathrm{~V}^{-1} \mathrm{~s}^{-1}$ [89-92]. It was also revealed that a dropcast film from $\mathrm{MCH}$ solution yielded the value of $\mu$ as $1 \times 10^{-4} \mathrm{~cm}^{2} \mathrm{~V}^{-1} \mathrm{~s}^{-1}$. Different self-assembly states strongly impact the charge transport capability (Table 1). The possible reason of larger electron mobility for xerogel from higher concentration solution $(4 \mathrm{mM})$ is that bundled fibers are efficiently formed in high concentration conditions and one-dimensional electron pathways are constructed. FPTRMC/TAS measurements were successfully carried out even in the gel state containing decane solvents. The electron mobility for the gel is smaller than its xerogel state. The thermal motion is the possible factors for the observed difference [93].

Table 1. Summary of observed maximum transient conductivity, charge carrier generation efficiency, and estimated mobility of cNDI-1.

\begin{tabular}{|c|c|c|c|}
\hline Samples & $\begin{array}{c}\phi \sum \mu \\
/ \mathrm{cm}^{2} \mathrm{~V}^{-1} \mathrm{~s}^{-1}\end{array}$ & $\phi$ & $\begin{array}{c}\sum \mu \\
/ \mathrm{cm}^{2} \mathrm{~V}^{-1} \mathrm{~s}^{-1}\end{array}$ \\
\hline xerogel $(0.1 \mathrm{mM})$ & $5.2 \times 10^{-6}$ & $7.9 \times 10^{-2}$ & $7 \times 10^{-5}$ \\
\hline dropcasted filma & $6.3 \times 10^{-6}$ & $6.4 \times 10^{-2}$ & $1 \times 10^{-4}$ \\
\hline gel (4 mM) & $4.5 \times 10^{-6}$ & $2.9 \times 10^{-2}$ & $2 \times 10^{-4}$ \\
\hline xerogel (4 mM) & $1.2 \times 10^{-5}$ & $3.3 \times 10^{-2}$ & $4 \times 10^{-4}$ \\
\hline
\end{tabular}

* From $0.1 \mathrm{mM}$ MCH solution.

\section{Conclusion}

The combined spectroscopy techniques of TRMC and TAS were successfully developed in the present study allowing us the method of fully-experimental non-contact assessment of electron mobility. Kinetics of photoconductivity and photo-generated radical anions were overlapped for the entire range of traces, suggesting the major contribution of electrons on the electronic conductivity on NDI stacks. The combined spectroscopy then determined the values of local scale mobility of electrons on NDI aggregates with different spatial arrangements via a variety of inter-molecular structural stabilization protocols. Although TRMC measurement deduces the mobility values from the small spatial perturbation of electrons within $\sim 0.1$ $\mathrm{nm}$, the values depend strongly on the spatial arrangement of NDI molecules. This suggests that the mobility determined by TRMC measurement reflects the dynamic disorder of the aggregates of $\pi$ conjugated systems. The successful experiments 
for gel states containing nonpolar solvents in the present work would extend the applicability of FPTRMC/TAS measurements to the assessment of electrical conductivity of 1) isolated molecules in solutions, 2) molecules in a variety of additives and quenchers, 3) molecules surrounded by a variety of media, 4) molecules under a variety of stimuli such as pressure, temperature, magnetic fields, etc. Particularly the dynamic modulation of the aggregated forms in solutions and the resulting conductivity changes will lead a remarkable impact on the related research field of supramolecular electronic materials.

\section{Acknowledgements}

S.S. and S.G. acknowledge and Japan Society for the Promotion of Science (JSPS) for the grants of Bilateral Joint Research Projects (DST/INT/JSPS/P-218/2016). This work was partly supported by a Grant-in-Aid for Scientific Research on Innovative Arias " $\pi$-System Figuration: Control of Electron and Structural Dynamism for Innovative Functions" (No. JP26102011 \& 15K21721) and Scientific Research (A) (No. 18H03918) from JSPS. T.S. acknowledges a Grant-in-Aid for Young Scientists (A) (No. 17H04880) from JSPS. S.G. thanks the Department of Science and Technology (DST), India, for funding through the SwarnaJayanti Fellowship Scheme (grant number DST/SJF/CSA01/2-14-15).

\section{References}

1. C. Wang, H. Dong, W. Hu, Y. Liu, and D. Zhu, Chem. Rev., 112 (2012) 2208.

2. L. Dou, Y. Liu, Z. Hong, G. Li, and Y. Yang, Chem. Rev., 115 (2015) 12633.

3. M. Saito, H. Shinokubo, and H. Sakurai, Mater. Chem. Front., 2 (2018) 635.

4. A. Tsumura, H. Koezuka, and T. Ando, Appl. Phys. Lett., 49 (1986) 1210.

5. H. Sirringhaus, P. J. Brown, R. H. Friend, M. M. Nielsen, K. Bechgaard, B. M. W. LangeveldVoss, A. J. H. Spiering, R. A. J. Janssen, E. W. Meijer, P. Herwig, and D. M. de Leeuw, Nature, 401 (1999) 685.

6. H. Scher and E. W. Montroll, Phys. Rev. B, 12 (1975) 2455.

7. P. M. Borsenberger, L. T. Pautmeier, and H. Bassler, Phys. Rev. B, 46 (1992) 12145.

8. N. T. Binh and L. Q. Mirth, Synth. Met., 58 (1993) 39.
9. M. A. Lampert and P. Mark, Current Injection in Solids, Academic Press, New York, 1970.

10. D. Hertel and H. Bässler, ChemPhysChem, 9 (2008) 666.

11. H. C. F. Martens, H. B. Brom, and P. W. M. Blom, Phys. Rev. B, 60 (1999) R8489.

12. H. C. F. Martens, J. N. Huiberts, and P. W. M. Blom, Appl. Phys. Lett., 77 (2000) 1852.

13. M. C. Beard, G. M. Turner, and C. A. Schmuttenmaer, J. Phys. Chem. B, 106 (2002) 7146.

14. J. B. Baxter and G. W. Guglietta, Anal. Chem., 83 (2011) 4342.

15. R. J. O. M. Hoofman, M. P. DeHaas, L. D. A. Siebbeles, and J. M. Warman, Nature, 392 (1998) 54.

16. P. G. Schouten, J. M. Warman, M. P. de Haas, M. A. Fox, and H. -L. Pan, Nature, 353 (1991) 736.

17. F. C. Grozema and L. D. A. Siebbeles, J. Phys. Chem. Lett., 2 (2011) 2951.

18. A. Acharya, S. Seki, A. Saeki, Y. Koizumi, and S. Tagawa, Chem. Phys. Lett., 404 (2005) 356.

19. A. Saeki, Y. Koizumi, T. Aida, and S. Seki, Acc. Chem. Res., 45 (2012) 1193.

20. S. Seki, A. Saeki, T. Sakurai, and D. Sakamaki, Phys. Chem. Chem. Phys., 16 (2014) 11093.

21. P. Prins, F. C. Grozema, J. M. Schins, S. Patil, U. Scherf, and L. D. A. Siebbeles, Phys. Rev. Lett., 96 (2006) 146601.

22. J. Terao, Y. Tanaka, S. Tsuda, N. Kambe, M. Taniguchi, T. Kawai, A. Saeki, and S. Seki, J. Am. Chem. Soc., 131 (2009) 18046.

23. J. Terao, A. Wadahama, A. Matono, T. Tada, S. Watanabe, S. Seki, T. Fujihara, and Y. Tsuji, Nat. Commun., 4 (2013) 1691.

24. K. Sugiyasu, Y. Honsho, R. M. Harrison, A. Sato, T. Yasuda, S. Seki, and M. Takeuchi, J. Am. Chem. Soc., 132 (2010) 14754.

25. C. Zhao, T. Sakurai, S. Yoneda, S. Seki, M. Sugimoto, C. Oki, M. Takeuchi, and K. Sugiyasu, Chem. Asian J., 11 (2016) 2284.

26. M. W. A. MacLean, T. Kitao, T. Suga, M. Mizuno, S. Seki, T. Uemura, and S. Kitagawa, Angew. Chem. Int. Ed., 55 (2016) 708.

27. T. Fukumatsu, A. Saeki, and S. Seki, Polym. Chem., 6 (2015) 5860.

28. T. Ohto, H. Masai, J. Terao, W. Matsuda, S. Seki, Y. Tsuji, and H. Tada, J. Phys. Chem. $C, 120$ (2016) 26637.

29. T. Sakurai, S. Yoneda, S. Sakaguchi, K. Kato, M. Takata, and S. Seki, Macromolecules, 50 (2017) 9265. 
30. Y. Yamamoto, T. Fukushima, Y. Suna, N. Ishii, A. Saeki, S. Seki, S. Tagawa, M. Taniguchi, T. Kawai, and T. Aida, Science, 314 (2006) 1761.

31. S. Yagai, Y. Nakano, S. Seki, A. Asano, T. Okubo, T. Isoshima, T. Karatsu, A. Kitamura, and Y. Kikkawa, Angew. Chem. Int. Ed., 49 (2010) 9990.

32. S. Patwardhan, S. Sengupta, L. D. A. Siebbeles, F. Würthner, and F. C. Grozema, J. Am. Chem. Soc., 134 (2012) 16147.

33. S. Prasanthkumar, S. Ghosh, V. C. Nair, A. Saeki, S. Seki, and A. Ajayaghosh, Angew. Chem. Int. Ed., 54 (2015) 946.

34. T. Mondal, T. Sakurai, S. Yoneda, S. Seki, and S. Ghosh, Macromolecules, 48 (2015) 879.

35. A. Takai, D. Sakamaki, S. Seki, Y. Matsushita, and M. Takeuchi, Chem. Eur. J., 22 (2016) 7385.

36. D. Basak, D. S. Pal, T. Sakurai, S. Yoneda, S. Seki, and S. Ghosh, Phys. Chem. Chem. Phys., 19 (2017) 31024.

37. T. Fukui, S. Kawai, S. Fujinuma, Y. Matsushita, T. Yasuda, T. Sakurai, S. Seki, M. Takeuchi, and K. Sugiyasu, Nat. Chem., 9 (2017) 493.

38. A. Sikder, J. Sarkar, T. Sakurai, S. Seki, and S. Ghosh, Nanoscale, 10 (2018) 3272.

39. G. Ghosh, M. Paul, T. Sakurai, W. Matsuda, S. Seki, and S. Ghosh, Chem. Eur. J., 24 (2018) 1938.

40. S. H. Mejías, J. López-Andarias, T. Sakurai, S. Yoneda, K. P. Erazo, S. Seki, C. Atienza, N. Martín, and A. L. Cortajarena, Chem. Sci., 7 (2016) 4842.

41. T. Amaya, S. Seki, T. Moriuchi, K. Nakamoto, T. Nakata, H. Sakane, A. Saeki, S. Tagawa, and T. Hirao, J. Am. Chem. Soc., 131 (2009) 408.

42. S. Sato, S. Seki, Y. Honsho, L. Wang, H. Nikawa, G. Luo, J. Lu, M. Haranaka, T. Tsuchiya, S. Nagase, and T. Akasaka, J. Am. Chem. Soc., 133 (2011) 2766.

43. B. M. Schmidt, S. Seki, B. Topolinski, K. Ohkubo, S. Fukuzumi, H. Sakurai, and D. Lentz, Angew. Chem. Int. Ed., 51 (2012) 11385.

44. Y. Tsutsui, T. Sakurai, S. Minami, K. Hirano, T. Satoh, W. Matsuda, K. Kato, M. Takata, M. Miura, and S. Seki, Phys. Chem. Chem. Phys., 17 (2015) 9624.

45. Y. Tsutsui, G. Schweicher, B. Chattopadhyay, T. Sakurai, J. B. Arlin, C. Ruzié, A. Aliev, A. Ciesielski, S. Colella, A. R. Kennedy, V. Lemaur, Y. Olivier, R. Hadji, L. Sanguinet, F. Castet, S. Osella, D. Dudenko, D. Beljonne, J.
Cornil, P. Samorì, S. Seki, and Y. H. Geerts, Adv. Mater., 7 (2016) 7106.

46. T. Higashino, T. Yamada, T. Sakurai, S. Seki, and H. Imahori, Angew. Chem. Int. Ed., $\mathbf{5 5}$ (2016) 12311.

47. T. Yoshida, D. Sakamaki, S. Seki, and H. Shinokubo, Chem. Commun., 53 (2017) 1112.

48. D. Sakamaki, H. Saeki, and S. Seki, Mater. Chem. Front., 2 (2018) 530.

49. S. Dalapati, M. Addicoat, S. Jin, T. Sakurai, J. Gao, H. Xu, S. Irle, S. Seki, and D. Jiang, Nat. Commun., 6 (2015) 7786.

50. J. Liu, W. Zhou, J. Liu, I. Howard, G. Kilibarda, S. Schlabach, D. Coupry, M. Addicoat, S. Yoneda, Y. Tsutsui, T. Sakurai, S. Seki, Z. Wang, P. Lindemann, E. Redel, T. Heine, and C. Wöll, Angew. Chem. Int. Ed., 54 (2015) 7441.

51. J. Liu, W. Zhou, J. Liu, Y. Fujimori, T. Higashino, H. Imahori, X. Jiang, J. Zhao, T. Sakurai, Y. Hattori, W. Matsuda, S. Seki, S. K. Garlapati, S. Dasgupta, E. Redel, L. Sun, and C. Wöll, J. Mater. Chem. A, 4 (2016) 12739.

52. M. J. W. Vermeulen, L. D. A. Siebbeles, J. M. Warman, A. Meisel, D. Neher, H.-G. Nothofer, and U. Scherf, Adv. Mater., 13 (2001) 1627.

53. Y. Honsho, T. Miyakai, T. Sakurai, A. Saeki, and S. Seki, Sci. Rep., 3 (2013) 3182.

54. W. Choi, T. Miyakai, T. Sakurai, A. Saeki, M. Yokoyama, and S. Seki, Appl. Phys. Lett., 105 (2014) 033302.

55. T. Sakurai, Y. Tsutsui, W. Choi, and S. Seki, Chem. Lett., 44 (2015) 1401.

56. W. Choi, Y. Tsutsui, T. Sakurai, and S. Seki, Appl. Phys. Lett., 110 (2017) 153303.

57. W. Choi, J. Inoue, Y. Tsutsui, T. Sakurai, S. Seki, Appl. Phys. Lett., 111 (2017) 203302.

58. W. Choi, Y. Tsutsui, T. Miyakai, T. Sakurai, and S. Seki, J. Phys. Conf. Series, 924 (2017) 012002 .

59. J. Inoue, Y. Tsutsui, W. Choi, K. Kubota, T. Sakurai, and S. Seki, ACS Omega, 2 (2017) 164.

60. A. Saeki, M. Tsuji, and S. Seki, Adv. Energy Mater., 1 (2011) 661.

61. A. Saeki, S. Yoshikawa, M. Tsuji, Y. Koizumi, M. Ide, C. Vijayakumar, and S. Seki, J. Am. Chem. Soc., 134 (2012) 19035.

62. R. Ihly, K. S. Mistry, A. J. Ferguson, T. T. Clikeman, B. W. Larson, O. Reid, O. V. Boltalina, S. H. Strauss, G. Rumbles, and J. L. Blackburn, Nat. Chem., 8 (2016) 603.

63. T. Umeyama, T. Miyata, A. C. Jakowetz, S Shibata, K. Kurotobi, T. Higashino, T. Koganezawa, M. Tsujimoto, S. Gélinas, W. 
Matsuda, S. Seki, R. H. Friend, and H. Imahori, Chem. Sci., 8 (2017) 181.

64. J. Baek, T. Umeyama, W. Choi, Y. Tsutsui, H. Yamada, S. Seki, and H. Imahori, Chem. Eur. J., 24 (2018) 1561.

65. R. R. Chance and C. L. Braun, J. Chem. Phys., 64 (1976) 3573.

66. C. L. Braun, J. Chem. Phys., 80 (1984) 4157.

67. R. Katoh and M. Kotani, J. Chem. Phys., 94 (1991) 5954.

68. D. Hertel and H. Bässler, ChemPhysChem, 9 (2008) 666.

69. A. Saeki, S. Seki, Y. Koizumi, and S. Tagawa, J. Photochem. Photobiol. A, 186 (2007) 158.

70. H. Kar, G. Ghosh, and S. Ghosh, Chem. Eur. J., 23 (2017) 10536.

71. H. E. Katz, A. J. Lovinger, J. Johnson, C. Kloc, T. Siegrist, W. Li, Y. Y. Lin, and A. Dodabalapur, Nature, 404 (2000) 478.

72. B. A. Jones, A. Facchetti, T. J. Marks, and M. R. Wasielewski, Chem. Mater., 19 (2007) 2703.

73. D. Shukla, S. F. Nelson, D. C. Freeman, M. Rajeswaran, W. G. Ahearn, D. M. Meyer, and J. T. Carey, Chem. Mater., 20 (2008) 7486.

74. S. V. Bhosale, C. H. Jani, and S. J. Langford, Chem. Soc. Rev., 37 (2008) 331.

75. S. P. Adiga and D. Shukla, J. Phys. Chem. C, 114 (2010) 2751.

76. S. L. Suraru and F. Würthner, Angew. Chem. Int. Ed., 53 (2014) 7428.

77. T. Sakurai, Y. Tsutsui, K. Kato, M. Takata, and S. Seki, J. Mater. Chem. C, 4 (2016) 1490.

78. M. Al Kobaisi, S. V. Bhosale, K. Latham, A. M. Raynor, and S. V. Bhosale, Chem. Rev., 116 (2016) 11685.

79. R. J. Chesterfield, J. C. McKeen, C. R. Newman, P. C. Ewbank, D. A. da Silva Filho, J.-L. Brédas, L. L. Miller, K. R. Mann, and C. D. Frisbie, J. Phys. Chem. B, 108 (2004) 19281.
80. C. Li and H. Wonneberger, Adv. Mater., 24 (2012) 613.

81. F. Würthner, C. R. Saha-Möller, B. Fimmel, S Ogi, P. Leowanawat, and D. Schmidt, Chem. Rev., 116 (2016) 962.

82. T. Sakurai, N. Orito, S. Nagano, K. Kato, M. Takata, and S. Seki, Mater. Chem. Front., 2 (2018) 718 .

83. D. S. Pal, H. Kar, and S. Ghosh, Chem. Eur. J., 22 (2016) 16872.

84. A. Das and S. Ghosh, Chem. Commun., 52 (2016) 6860.

85. H. Kar, D. W. Gehrig, N. K. Allampally, G. Fernández, F. Laquai, and S. Ghosh, Chem. Sci., 7 (2016) 1115.

86. P. Rajdev, S. Chakraborty, M. Schmutz, P. Mesini, and S. Ghosh, Langmuir, 33 (2017) 4789.

87. M. R. Ajayakumar, D. Asthana, and P. Mukhopadhyay, Org. Lett., 14 (2012) 4822.

88. S. Chopin, F. Chaignon, E. Blart, and F. Odobel, J. Mater. Chem., 17 (2007) 4139.

89. C. W. Struijk, A. B. Sieval, J. E. J. Dakhorst, M. Van Dijk, R. B. M. Koehorst, H. Donker, T. J. Schaafsma, S. J. Picken, A. M. Van De Craats, J. M. Warman, H. Zuilhof, and E. J. R. Sudhlter, J. Am. Chem. Soc., 122 (2000) 11057.

90. F. C. Grozema, L. D. A. Siebbeles, J. M. Warman, S. Seki, S. Tagawa, and U. Scherf, Adv. Mater., 14 (2002) 228.

91. H. Yokoi, Y. Hiraoka, S. Hiroto, D. Sakamaki, S. Seki, and H Shinokubo, Nat. Commun., 6 (2015) 8215.

92. S. Sato, H. Nikawa, S. Seki, L. Wang, G. Luo, J. Lu, M. Haranaka, T. Tsuchiya, S. Nagase, and T. Akasaka, Angew. Chem. Int. Ed., 51 (2012) 1589.

93. S. Illig, A. S. Eggeman, A. Troisi, L. Jiang, C. Warwick, M. Nikolka, G. Schweicher, S. G. Yeates, Y. H. Geerts, J. E. Anthony, and H. Sirringhaus, Nat. Commun., 7 (2016) 1. 